Maarten P. de Boer, ${ }^{1}$ Norman F. Smith, ${ }^{1}$ Nathan D. Masters, ${ }^{1}$ Michael B. Sinclair ${ }^{1}$ and Emily J. Pryputniewicz ${ }^{2}$

\title{
Integrated platform for testing MEMS mechanical properties at the wafer scale by the IMaP methodology
}

REFERENCE: de Boer, M. P., Smith, N. F., Masters, N. D., Sinclair M. B., Pryputniewicz, E. J., "Integrated platform for testing MEMS mechanical properties at the wafer scale by the IMaP methodolgy" Mechanical Properties of Structural Films, STP No. 1413 (S. B. Brown and C. L. Muhlstein), Eds., American Society for Testing and Materials, West Conshohocken, PA, 2000.

\begin{abstract}
A new instrument to accurately and verifiably measure mechanical properties across an entire MEMS wafer is under development. We have modified the optics on a conventional microelectronics probe station to enable three-dimensional imaging while maintaining the full working distance of a long working distance objective. This allows standard probes or probe cards to be used. We have proceeded to map out mechanical properties along a wafer column by the Interferometry for Material Property Measurement (IMaP) methodology. From interferograms of simple actuated cantilevers, out-of-plane deflection profiles at the nanometer scale are obtained. These are analyzed by integrated routines that extract basic mechanical properties such as Young's modulus and cantilever curvature. Non-idealities such as support post compliance and beam take off angle are simultaneously quantified. Verifiability is achieved by comparing properties at multiple voltages. When the non-idealities are properly taken into account, we find that Young's modulus $E \sim 161 \mathrm{GPa}$ for polysilicon and is independent of wafer position. However, curvature and residual stress data correlate with wafer position.
\end{abstract}

KEYWORDS: mechanical property characterization, wafer scale, long working distance interferometry, software integration, MEMS metrology

\section{Introduction}

Fabrication of microelectromechanical systems (MEMS) devices for a variety of applications including optical switches and displays, microrelays, accelerometers, gyroscopes, image correctors, printheads, flow sensors, and medical devices is currently of great interest. MEMS are fabricated in a fashion similar to microelectronics in the integrated circuit (IC) industry. Free standing structures are created at the end of the process flow by removing the oxide matrix surrounding thin film structural members. Polycrystalline silicon (polysilicon) is to date the most successful MEMS material

\footnotetext{
${ }^{1}$ Sandia National Laboratories, PO Box 5800, Albuquerque, NM, 87185

${ }^{2}$ Worcestor Polytechnic Institute, Worcester, MA, 01609
} 


\section{DISCLAIMER}

This report was prepared as an account of work sponsored by an agency of the United States Government. Neither the United States Government nor any agency thereof, nor any of their employees, make any warranty, express or implied, or assumes any legal liability or responsibility for the accuracy, completeness, or usefulness of any information, apparatus, product, or process disclosed, or represents that its use would not infringe privately owned rights. Reference herein to any specific commercial product, process, or service by trade name, trademark, manufacturer, or otherwise does not necessarily constitute or imply its endorsement, recommendation, or favoring by the United States Government or any agency thereof. The views and opinions of authors expressed herein do not necessarily state or reflect those of the United States Government or any agency thereof. 


\section{DISCLAIMER}

Portions of this document may be illegible in electronic image products. Images are produced from the best available original document. 
because many requirements can be satisfied simultaneously [1]. Other structural materials such as aluminum [2], silicon carbide [3] and "amorphous diamond" [1] are in use or being explored.

Thin film techniques such as chemical vapor deposition, sputtering or pulsed laser ablation are used to form such structural materials. For volume production, the same MEMS device will be fabricated repeatedly over the surface of a wafer, usually $15 \mathrm{~cm}$ or more in diameter. Typically there are 50 or more identical die sites. The microstructure of the resulting films from any of these deposition methods can exhibit cross-wafer nonuniformities resulting in variations of thickness, height and perhaps residual stress as well as stress gradient or even modulus across the wafer. Both mechanical and surface properties must be sufficiently well controlled to guarantee that the intended design function of the MEMS device is met across the wafer. For example, the resonant frequency of a comb drive is sensitive to small variations in residual stress. Also, highly curved comb drive fingers or suspensions (caused by stress gradient) will result in device malfunction. Furthermore, surface properties such as adhesion and friction are very sensitive to processing, and may exhibit cross-wafer non-uniformity as well. Poor control of surface properties may result in failure of devices that allow contact or sliding of surfaces. Also, multiple levels of structural material are now being fabricated. It is important to determine mechanical and surface properties of each of these levels. No MEMS metrology instrument exists today that (1) is highly sensitive to both mechanical and surface properties and (2) allows rapid property extraction across an entire wafer.

We have been investigating

Test Structure $\quad$ Properties

(a)

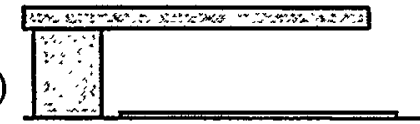

(b)

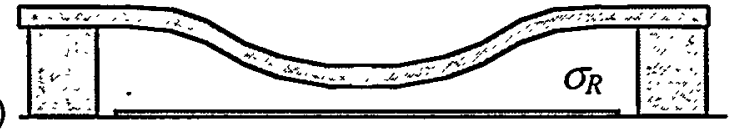

(c)

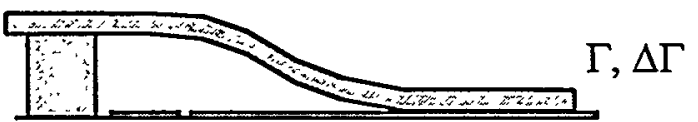

(d)

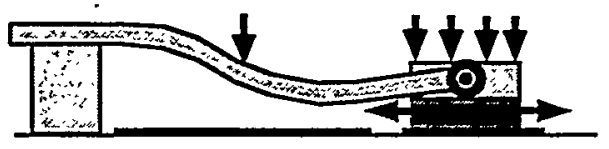

(e)

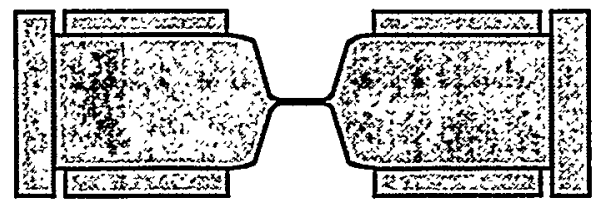

Fig. 1 Suite of test devices to measure mechanical and surface properties in MEMS $t, g, \theta_{o}, \kappa$,

$E, \beta$

$\mu$

$\sigma_{f}$ die site, important because more area is then available to build the intended devices. Electrostatic loading is used in $\mathrm{IMaP}$ because this is the primary actuation means in MEMS. Leakage currents that adversely affect test

\section{TEOEREE}

NOY $2 \& 2000$ 
structures can serve as early diagnosis for failure of real MEMS devices.

The test structures we have been investigating are schematically represented in Fig. 1. Properties including thickness $t$, gap $g$, takeoff angle $\theta_{i}$, curvature $\kappa$, support post compliance $\beta$ and Young's modulus $E$ can be obtained from cantilevers [4-6], as seen in Fig. 1(a). Deflections of actuated fixed-fixed beams provide a sensitive measure of residual stress $\sigma_{R}$ [4,5] (Fig. 1(b)). Cantilevers are also very sensitive to adhesion to the substrate due to capillary or van der Waals forces (Fig. 1(c)), and can be used to accurately measure adhesion [7,8] as well as adhesion hysteresis [9]. Also, small area devices to monitor coefficient of friction $\mu[10,13]$ (Fig. 1(d)) and fracture strength $\sigma_{f}$ [11] (Fig. 1(e)) have been designed, monitored by interferometry, and analyzed.

Although we have shown that all these properties can be well monitored by integrating nm-scale deflection data with computer-based models, two factors limit the ability to apply these techniques at the wafer scale. First, it is convenient to use conventional microelectronics probe stations that are manufactured to align wafers and move from one die site to the next for this purpose. The electrical probes or probe cards for such systems usually require 1 to $2 \mathrm{~cm}$ of free space between the wafer surface and the microscope objective. However, the free working distance of commercially available interferometers is generally small, less than $1 \mathrm{~cm}$, because an attachment enabling the interferometry is usually placed between the objective and the sample surface. Second, analysis routines to extract material properties from deflection data of actuated beams are slow.

We demonstrate in this paper that both of these problems can be surmounted. The optics on a standard probe station microscope have been modified to allow interferometry with no loss of free working distance. This microscope has been directly mounted on a conventional microelectronics probe station manufactured to align wafers and move from one die site to the next, allowing wafer scale testing. Also, we have significantly enhanced the speed of material property determination by writing software routines that rapidly converge on unique solutions. We show testing results of actuated cantilevers and fixed-fixed beams from different die sites on a column of a wafer. While the results here are for polysilicon, the technique is also applicable to other MEMS materials.

\section{Long free working distance interferometry}

In most interferometric microscopes, an incoherent source with a coherence length of $\sim 10 \mu \mathrm{m}$ is used for illumination. The key requirement for incoherent source interferometry is that the optical path lengths from the beam splitter to the reference surface and to the sample must be the same to within a few microns. This is usually achieved by placing the beam splitter and reference surface under the microscope objective in either a Michelson or Mirau realization, and comes at the expense of free working distance. In work to show the feasibility of the IMaP approach [4-11], we have previously used a Michelson attachment with a five millimeter free working distance. Test structures were actuated on individual chips with $3 \mathrm{~mm}$ high electrical probes. Such an approach is awkward at the wafer scale. One method to gain the full free working distance of an objective is to use a second objective matched to the first. However, long working distance objectives required for wafer scale probing were not suitably matched for this approach to be effective. 

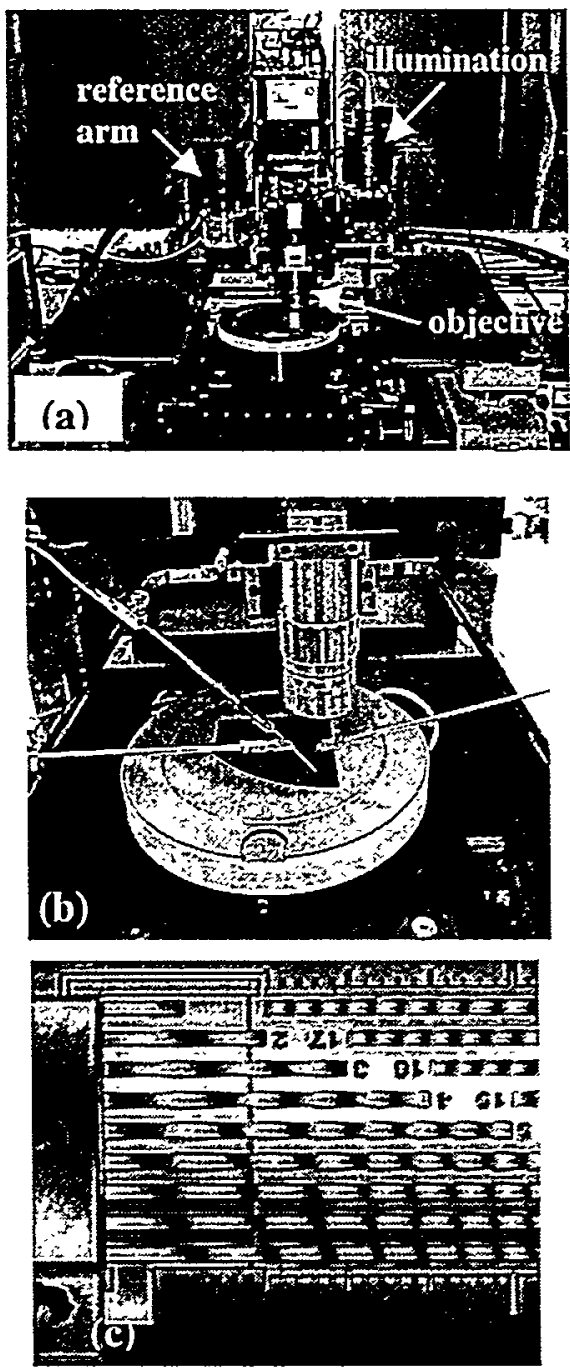

Fig. 2 (a) Modified

microscope on probe station, (b) closeup of objective with

$2.5 \mathrm{~cm}$ free working distance,

(c) interferogram of cantilever beam array
If the illumination is replaced by a coherent source, the path length requirement is alleviated. As seen in Fig. 2, it is then possible to modify a commercial microprober optical microscope to obtain interferometric imaging. Details of the implementation will be published elsewhere. In Fig. 2(a), the beam splitter behind the objective is used for the reference wave, and the optical path lengths to the sample and to the reference surface are quite different. The illumination source is a $532 \mathrm{~nm}$ (green light) diode pumped, frequency doubled Nd:YAG laser, with a coherence length of greater than $100 \mathrm{~m}$. Green light is chosen because polysilicon has low transmission at this wavelength, and because objective aberrations are minimized at the center of the visible spectrum. As seen in Fig. 2(b), the full free working distance of the original microscope objective $(-2.5 \mathrm{~cm})$ is maintained, and off-the-shelf commercial probes fit underneath easily. A good quality inteferometric image of cantilever beam test structures is seen in Fig. 2(c). Vibration has been reduced by placing the unit on an isolation table. Because the reference surface is mounted on a voltage controlled piezoelectric crystal, its z-height can be finely tuned.

This design realization has numerous advantages over incoherent light interferometry. First, it is possible to obtain interferometry using different microscope objectives with only minor adjustment of the reference surface. Although $5 \mathrm{X}$ and $10 \mathrm{X}$ objectives were used in this work, good quality images of small structures have also been obtained with a $50 \mathrm{X}$ objective at $1.3 \mathrm{~cm}$ free working distance. Also, the microscope has an

internal zoom, giving a continuously adjustable magnification range of greater than twenty without changing objectives. Second, with this implementation, it is possible to image through a glass window, important for applications in which the device is in vacuum or in a sealed package. Third, besides monitoring simple test structures as in this work, this capability will also be very useful for 3-dimensional imaging of full-scale micromachined devices at the wafer level. Fourth, the illumination is very bright, which is advantageous in high frame rate or stroboscopic applications. 


\section{Algorithm \& software to determine cantilever properties}

To test the effectiveness of the new system, we have measured cantilever and fixed-fixed beam properties along a column of a wafer. In Fig. 3, the step-by-step procedure to determine properties is shown, and in Fig. 4, the cantilever and its nonidealities are schematically represented. The algorithm to determine cantilever properties has been detailed [6] and, along with software enhancements, is briefly described next.

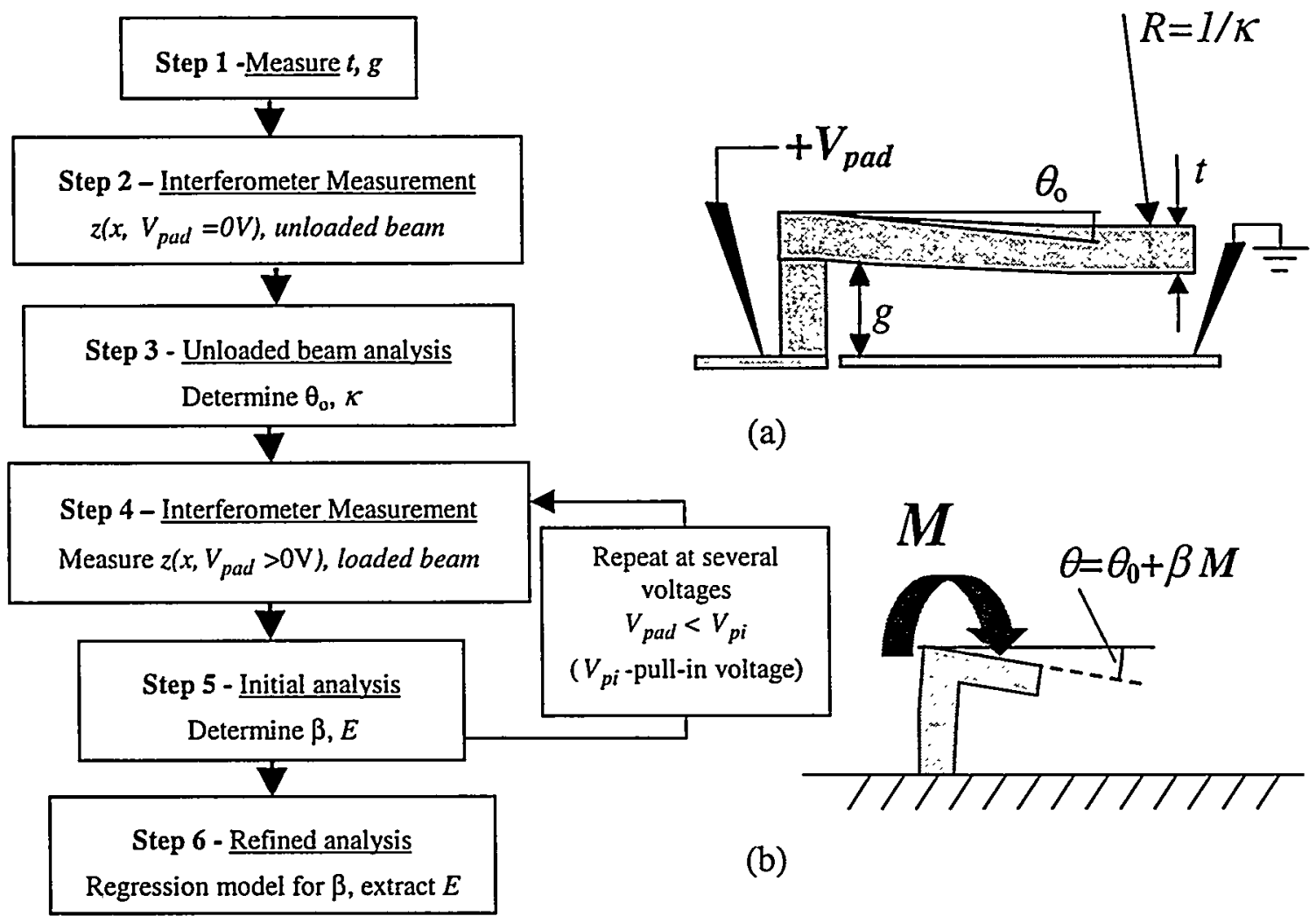

Fig. 3 step-by-step procedure to determine cantilever properties.

Fig. 4 (a) Cantilever parameters $t, g, \theta_{o}$ and $R=1 / \kappa$, (b) support post model

Three software routines have been written to enable rapid property determination, as portrayed in Fig. 5. Interferograms of unloaded beams are taken using an image analyzer program called "LineProfile Tool" per step 2 of Fig. 3. Within this program, reference points indicating the beginning and end of the beam, as well as reference points indicating where the linescan begins and ends, are superposed on the image. The linescan, containing fringe information as a function of pixel position, along with the reference information and the beam length, are stored in a file for further processing by a program called "Deflection Calculator". It converts the linescan information into pixel by pixel $z$-deflection data with near $\mathrm{nm}$ resolution, and also converts pixel data into $x$ position data. A program called "BeamPROPS" operates on the deflection data to extract properties. BeamPROPS calculates model deflections by the finite difference method. For unloaded beams, it accounts for the non-idealities $\theta_{o}$ and $\kappa$, and for the small effect of gravity. It determines the properties per step 3 of Fig. 3 by adjusting the model deflections until the error between the data and the model deflections are minimized 


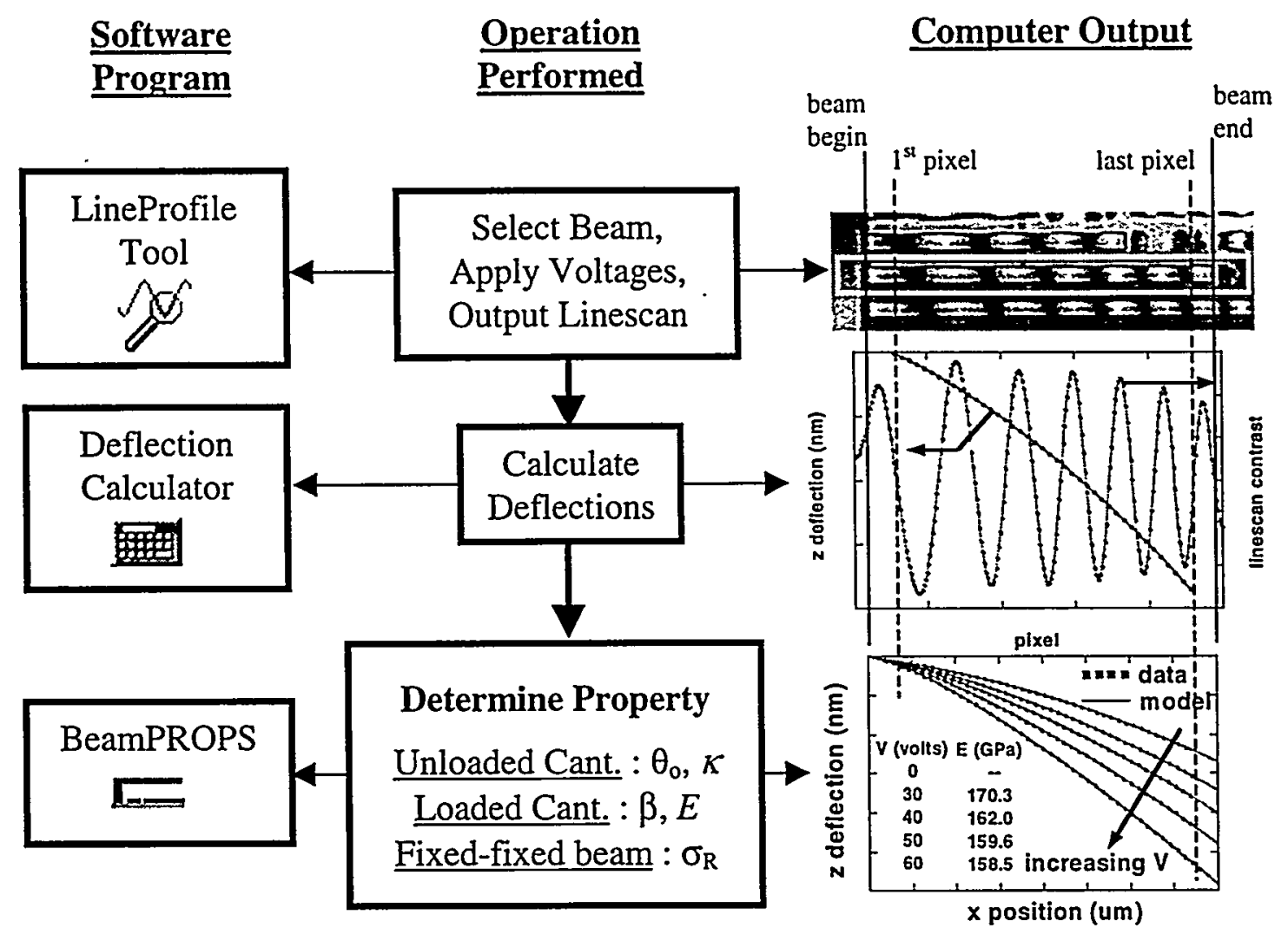

Fig. 5 Overview of algorithms to determine properties of beams

using a quasi-Newton search algorithm. For example, a comparison of experimental and model deflections of unloaded cantilevers along a wafer column are shown in Fig. 6. These deflections are well described by the equation

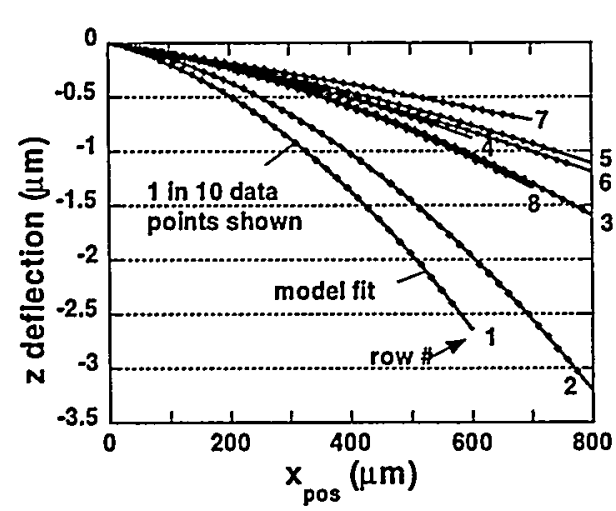

Fig. 6 Deflection profiles of unloaded cantilevers.

$$
z(x)=\theta_{o} x+\kappa \frac{x^{2}}{2}
$$

where $z(x)$ is positive for deflections away from the substrate. Typical root mean square (rms) errors between the data and the model are $\sim 2$ $\mathrm{nm} /$ pixel. The resolution for $\theta_{o}$ is approximately $50 \mu \mathrm{rad}$, while for $\kappa$ it is approximately $1 \mathrm{~m}^{-1}$, corresponding to $\sim 0.15 \mathrm{MPa} / \mu \mathrm{m}$ stress gradient resolution (note that stress gradient $=E \kappa$ ).

Once $\theta_{o}$ and $\kappa$ are known, a similar analysis procedure is performed by BeamPROPS on electrostatically loaded beams to find $E$ and $\theta$

per steps 4 and 5 of Fig. 3. The value of $K$ is held fixed, and model deflections are calculated for a given value of $E$ and loaded beam takeoff angle $\theta$. Electrostatic loading with a fringing field correction is incorporated in the finite difference model calculations. 
The quasi-Newton search algorithm travels through $(E, \theta)$ space to rapidly determine an optimum fit between the measured and modeled data, typically within five seconds. For a given cantilever, values of loaded beam takeoff angle $\theta$ and $E$ are determined at each voltage loading. Non-systematic errors affect these values. Therefore, the slope in a regression model in which $\theta$ is plotted against the calculated moment $M$ is used to determine a value for $\beta$, according to the equation

$\theta=\theta_{o}+\beta M$,

as shown in Fig 4. Using the regression model for $\beta$, The data is re-analyzed by BeamPROPS to determine the best values for $E$, per step 6 of Fig. 3 . An example of the optimum fit to the measured data, as well as the associated values for $E$, are shown in the bottom right hand corner of Fig. 5. Note that at larger voltages, where $E$ exerts its maximum effect on flexures, the values of $E$ are repeatable. In the wafer column data below, values of $E$ at each die site are averaged for several voltage loadings and several beams.

The deflection profiles of unloaded fixed-fixed beams of $1000 \mu \mathrm{m}$ length were measured and were well represented by a sinusoidal shape, indicating that the beams were buckled in compression. The residual stress $\sigma_{R}$ (due to average residual stress through the thickness of the film) was calculated from the Euler buckling result

$\sigma_{R}=-E \frac{\pi^{2}}{12}\left(\frac{3 A^{2}+4 t^{2}}{L^{2}}\right)$

where $L$ is the length of the fixed-fixed beam and $A$ is the buckled beam amplitude, and the minus sign is for compression. The equation assumes that the beam is well into the post-buckling regime, which is very reasonable for these long beams. The effect of stress gradient on the calculated result is small, and the resolution of the measurement with respect to $\sigma_{R}$ is $1 \mathrm{MPa}$ or better. A value of $E=161 \mathrm{GPa}$ was assumed in the calculations.

\section{Results and discussion}

Diagnostic test structures were laid out and included on each die site along with the standard MEMS devices. A lot was fabricated according to the standard five level process flow [1], in which four independent structural layers of polysilicon are produced. After processing, structures were freed by a standard "release" process, and thickness of the polysilicon layers was measured using a profilometer to $\sim 20 \mathrm{~nm}$ accuracy. The wafer was then loaded onto the testbed described above. Cantilevers tested were 300 to $700 \mu \mathrm{m}$ in length, while the fixed-fixed beams were $1000 \mu \mathrm{m}$ in length.

Fig. 7 shows testing results of mechanical properties along a column of die sites as represented in Fig. 6(a), for the third level of polysilicon, "Poly 3". Fig. 6(b) shows tight control of thickness, with $t=2.34+/-0.02 \mu \mathrm{m}$. Gap height $g$ was measured interferometrically to $\sim 20 \mathrm{~nm}$ accuracy on cantilevers actuated into contact with the substrate. Poly 3 gap height $g$ is not as well controlled as thickness, with values from 
(a)

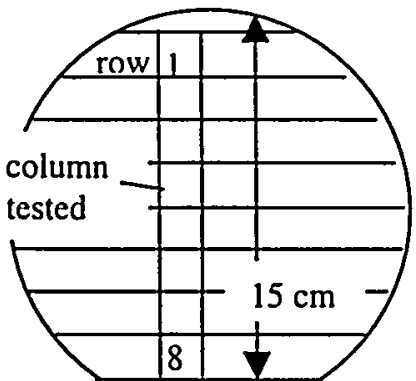

(b)

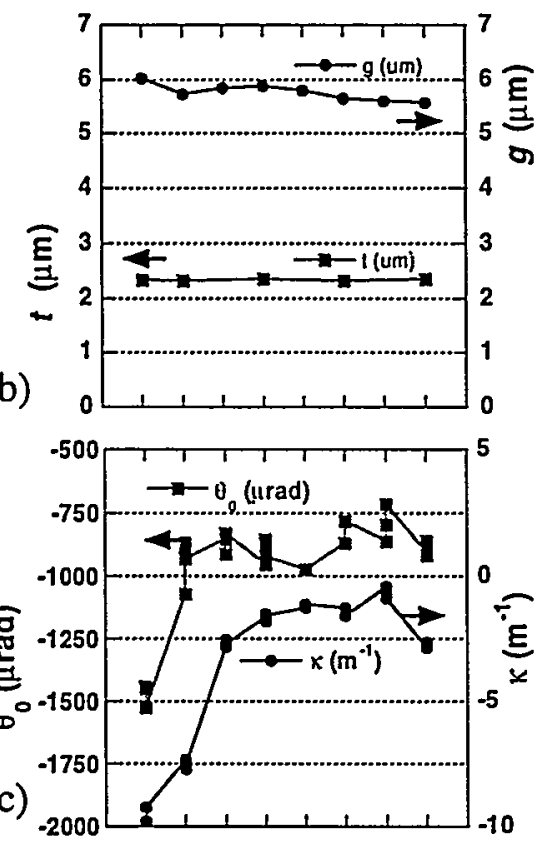

(d)

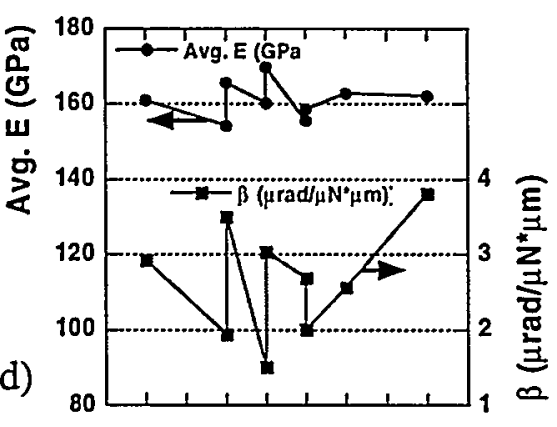

(e)

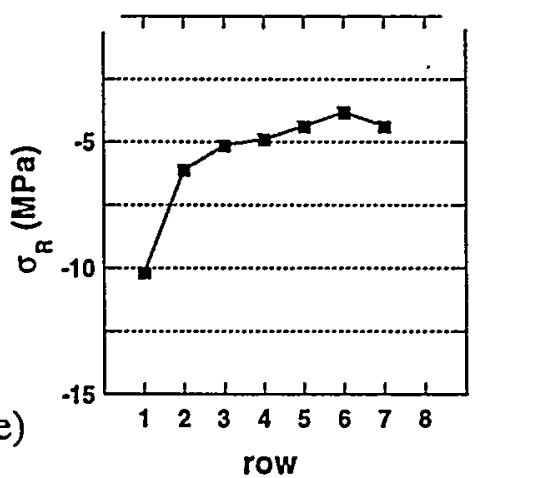

Fig. 7 Column map for materials properties
(a) wafer, (b) $t, g$,
(c) $\theta_{0}, \kappa$,
(d) $E, \beta$,
(e) $\sigma_{R}$

5.53 to $6.03 \mu \mathrm{m}$. This variation across the wafer is reasonable given the chemical mechanical polishing (CMP) process of the oxide layer underlying Poly 3 . It is important to measure the geometrical properties $t$ and $g$ at this accuracy level $(-20 \mathrm{~nm})$ on each individual device, because the subsequent analysis to determine $E$ depends strongly on these values.

Using Eq. (1), the optimum values of unloaded cantilever takeoff angle $\theta_{o}$ and $\kappa$ reflecting the data of Fig. 6 are shown in Fig. 7(c). It is seen that $\theta_{o}$ is negative but relatively constant across the wafer, with values of 750 to $-1000 \mu$ radians. The negative values of $\theta_{o}$ probably result from oxide incorporation within the support post structure. After release, the highly compressive oxide is no longer constrained, and induces the support to pivot. This is undesirable because it forces long cantilevers to touch the substrate, reducing the effectiveness of the test structures, but can be improved with a better support post layout. Values of $\kappa$ from -1 to $2.5 \mathrm{~m}^{-1}$ (corresponding to stress gradient values of -0.16 to $-0.4 \mathrm{MPa} / \mu \mathrm{m}$ ) are quite low for MEMS devices. Note in row 1 that $\theta_{o}$ drops sharply to $-1500 \mu \mathrm{rad}$ and also that $\kappa$ decreases significantly to $-10 \mathrm{~m}^{-1}$. The change in $\kappa$ may be due to a subtle microstructural feature or trace contaminant such as the oxygen level being different in the Poly 3 layer at this die location relative to the rest of the wafer. This in turn may be responsible for the sharp drop in $\theta_{o}$. Clearly, both negative $\theta_{o}$ and negative $K$ are undesirable for MEMS devices that often contain long structural elements assumed to be flat over the substrate.

In Fig. 7(d), the values of $E$ are seen to be relatively constant across the substrate. This is to be expected because it is unlikely that the subtle changes in stress gradient at the sub $\mathrm{MPa} / \mu \mathrm{m}$ level (or the changes in $\sigma_{R}$ at $<10 \mathrm{MPa}$, see below) will affect the bulk modulus of the film of $\sim 160 \mathrm{GPa}$. However, it is important to note that given the highly non-linear mechanics of the electrostatically actuated beam, this result can only be achieved because $t, g, \theta_{o}$ and $\kappa$ were measured on the same individual cantilevers. The average value of $\beta \sim 2.5 \mu \mathrm{rad} /\left(\mu \mathrm{N}^{*} \mu \mathrm{m}\right)$ compares well with finite element modeling of similar geometries, where $\beta$ values of 2.47 to $2.73 \mu \mathrm{rad} /\left(\mu \mathrm{N}^{*} \mu \mathrm{m}\right) \quad$ were determined [6]. However, $\beta$ varies significantly across the wafer without a trend. Because its value is 
dominated by the polysilicon thickness which was well controlled, it would not be expected that $\beta$ would vary to this degree. We are investigating the resolution of the algorithms on $\beta$.

The values of residual stress $\sigma_{R}$ correlate well with curvature $\kappa$, as seen in Fig. $7(\mathrm{e})$. This is not necessarily expected, but is perhaps not surprising because $K$ is caused by the gradient in $\sigma_{R}$ through the thickness of the film.

\section{Summary and conclusions}

We have constructed a long working distance interferometer to enable three dimensional visualization of MEMS devices and test structures on a standard microelectronics probing station. This enables nm-scale deflection profiles of test structures to be acquired across a wafer. We have also written software routines that enable rapid comparison of deflection data with test structure models, allowing fundamental property extraction. In the course of obtaining test structure properties along a wafer column, the new instrument has been validated to work well. These property measurements will prove invaluable for process control and yield enhancement in MEMS and for understanding limitations of MEMS device design. Future work will include extending this technique to measurement of other polysilicon layers in our MEMS process, as well as to surface properties such as adhesion and friction.

\section{Acknowledgments}

We would like to acknowledge the staff in the microelectronics development lab at Sandia National Laboratories for fabrication of the test structures. Sandia is a multiprogram laboratory operated by Sandia Corporation, a Lockheed Martin Company, for the United States Department of Energy under Contract DE-AC04-94AL85000.

\section{References}

[1] J. J. Sniegowski and M. P. de Boer, IC-compatible polysilicon surface micromachining, Annu. Rev. Mater. Sci. 30297 (2000).

[2] P. F. Vankessel, L. J. Hornbeck, R. E. Meier and M. R. Douglass, MEMS based projection display, Proc. IEEE 86 (8) 1687 (1998).

[3] M. Mehregany and C. A. Zorman, SiC MEMS: opportunities and challenges for applications in harsh environments, Thin Solid Films 356518 (1999).

[4] B. D. Jensen, M. P. de Boer and S. L. Miller, IMaP: Interferometry for materials property evaluation in MEMS, MSM 99, San Juan, Puerto Rico, 1999, pp. 206-209.

[5] B. D. Jensen, M. P. de Boer and F. Bitsie, Interferometric measurement for improved understanding of boundary effects in micromachined beams, Proceedings of the SPIE, Materials and Device Characterization in Micromachining II, Santa Clara, CA, 1999, pp. 61-72.

[6] B. D. Jensen, M. P. de Boer, N. D. Masters, F. Bitsie and D. A. LaVan, Precise measurement of Young's modulus in the presence of test structure non-idealities, J. MEMS submitted (2000).

[7] M. P. de Boer and T. A. Michalske, Accurate method for determining adhesion of cantilever beams, J. Appl. Phys. 86 (2) 817 (1999). 
[8] M. P. de Boer, P. J. Clews, B. K. Smith and T. A. Michalske, Adhesion of polysilicon microbeams in controlled humidity ambients, Mater. Res. Soc. Proc. 518131 (1998).

[9] M. P. de Boer, J. A. Knapp, T. A. Michalske, U. Srinivasan and R. Maboudian, Adhesion hysteresis of silane coated microcantilevers, Acta Mater. in press (2000).

[10] M. P. de Boer, J. M. Redmond and T. A. Michalske, A hinged-pad test structure for sliding friction measurement in micromachinig, Proceedings of the SPIE, Materials and Device Characterization in Micromachining, Santa Clara, CA, 1998, pp. 241250.

[11] M. P. de Boer, B. D. Jensen and F. Bitsie, A small area in-situ MEMS test structure to measure fracture strength by electrostatic probing, Proceedings of the SPIE, Materials and Device Characterization in Micromachining II, Santa Clara, CA, 1999, pp. 97-103.

[12] M. P. de Boer, B. D. Jensen, S. L. Miller, N. F. Smith and M. B. Sinclair, Automated test system to accurately measure and verify mechanical and surface properties in MEMS, patent pending (2000).

[13] B. T. Crozier, M. P. de Boer, J. M. Redmond, D. F. Bahr and T. A. Michalske, Friction measurement in MEMS using a new test structure, Materials Research Society Symposium Proceedings, Boston, MA, 2000, pp. 129-134. 